\title{
Rainbow Matchings of Size $\delta(G)$ in Properly Edge-Colored Graphs
}

\author{
Jennifer Diemunsch ${ }^{1,4} \quad$ Michael Ferrara ${ }^{1,5} \quad{\text { Allan } \text { Lo }^{2,6}}^{2,6}$ \\ Casey Moffatt ${ }^{1} \quad$ Florian Pfender ${ }^{3} \quad$ Paul S. Wenger ${ }^{1}$
}

Submitted: Dec 21, 2011; Accepted: Jun 16, 2012; Published: Jun 28, 2012

\begin{abstract}
A rainbow matching in an edge-colored graph is a matching in which all the edges have distinct colors. Wang asked if there is a function $f(\delta)$ such that a properly edgecolored graph $G$ with minimum degree $\delta$ and order at least $f(\delta)$ must have a rainbow matching of size $\delta$. We answer this question in the affirmative; an extremal approach yields that $f(\delta)=98 \delta / 23<4.27 \delta$ suffices. Furthermore, we give an $O\left(\delta(G)|V(G)|^{2}\right)$ time algorithm that generates such a matching in a properly edge-colored graph of order at least $6.5 \delta$.
\end{abstract}

Keywords: Rainbow matching, properly edge-colored graphs

\section{Introduction}

All graphs under consideration in this paper are simple, and we let $\delta(G)$ and $\Delta(G)$ denote the minimum and maximum degree of a graph $G$, respectively. In this paper, we consider edge-colored graphs and let $c(u v)$ denote the color of the edge $u v$. An edge coloring of a graph is proper if the colors on edges sharing an endpoint are distinct. An edge-colored graph is rainbow if all edges have distinct colors. Rainbow matchings are of particular interest given their connection to transversals of Latin squares: each Latin square can be converted to a properly edge-colored complete bipartite graph, and a transversal of the Latin square is a rainbow perfect matching in the graph. Ryser's conjecture [9] that every Latin square of odd order has a transversal can be seen as the beginning of the study of rainbow

\footnotetext{
${ }^{1}$ Dept. of Mathematical and Statistical Sciences, Univ. of Colorado Denver, Denver, CO; email addresses: jennifer.diemunsch@ucdenver.edu, michael.ferrara@ucdenver.edu, casey.moffatt@ucdenver.edu, paul. wenger@ucdenver. edu.

${ }^{2}$ School of Mathematics, University of Birmingham, Birmingham B15 2TT, UK, s.a.lo@bham.ac.uk

${ }^{3}$ Institut für Mathematik, Univ. Rostock, Rostock, Germany; Florian.Pfender@uni-rostock.de.

${ }^{4}$ Research supported in part by UCD GK12 Transforming Experiences Project, NSF award \# 0742434.

${ }^{5}$ Research supported in part by Simons Foundation Collaboration Grant \# 206692.

${ }^{6}$ Research supported in part by Eurupean Research Council Grant \# 258345.
} 
matchings. Stein [10] later conjectured that every Latin square of order $n$ has a transversal of size $n-1$; equivalently every proper edge-coloring $K_{n, n}$ has a rainbow matching of size $n-1$. The connection between Latin transversals and rainbow matchings in $K_{n, n}$ has inspired additional interest in the study of rainbow matchings in triangle-free graphs. A thorough survey of rainbow matching and other rainbow subgraphs in edge-colored graphs appears in [6].

Several results have been attained for rainbow matchings in arbitrarily edge-colored graphs. The color degree of a vertex $v$ in an edge-colored graph $G$, written $\hat{d}(v)$, is the number of distinct colors on edges incident to $v$. We let $\hat{\delta}(G)$ denote the minimum color degree among the vertices in $G$. Wang and $\mathrm{Li}$ [12] proved that every edge-colored graph $G$ contains a rainbow matching of size at least $\left[\frac{5 \hat{\delta}(G)-3}{12}\right\rceil$, and conjectured that a rainbow matching of size $\lceil\hat{\delta}(G) / 2\rceil$ exists if $\hat{\delta}(G) \geqslant 4$. LeSaulnier et al. [8] then proved that every edge-colored graph $G$ contains a rainbow matching of size $\lfloor\hat{\delta}(G) / 2\rfloor$. Finally, Kostochka and Yancey [5] proved the conjecture of Wang and $\mathrm{Li}$ in full, and also that triangle-free graphs have rainbow matchings of size $\lceil 2 \hat{\delta}(G) / 3\rceil$.

Since the edge-colored graphs generated by Latin squares are properly edge-colored, it is of interest to consider rainbow matchings in properly edge-colored graphs. In this direction, LeSaulnier et al. proved that a properly edge-colored graph $G$ satisfying $|V(G)| \neq \delta(G)+2$ that is not $K_{4}$ has a rainbow matching of size $\lceil\delta(G) / 2\rceil$. Wang then asked if there is a function $f$ such that a properly edge-colored graph $G$ with minimum degree $\delta$ and order at least $f(\delta)$ must contain a rainbow matching of size $\delta$ [11]. As a first step towards answering this question, Wang showed that a graph $G$ with order at least $8 \delta / 5$ has a rainbow matching of size $\lfloor 3 \delta(G) / 5\rfloor$.

Since there are $n \times n$ Latin squares with no transversals when $n$ is even (see [1, 14]), for such a function $f$ it is clear that $f(\delta)>2 \delta$ when $\delta$ is even. Furthermore, since maximum matchings in $K_{\delta, n-\delta}$ have only $\delta$ edges (provided $n \geqslant 2 \delta$ ), there is no function for the order of $G$ depending on $\delta(G)$ that can guarantee a rainbow matching of size greater than $\delta(G)$.

In this paper we answer Wang's question from [11] in the affirmative, proving that a linear number of vertices in terms of the minimum degree suffices.

Theorem 1. If $G$ is a properly edge-colored graph satisfying $|V(G)| \geqslant 98 \delta(G) / 23$, then $G$ contains a rainbow matching of size $\delta(G)$.

Independently, Wang, Zhang, and Liu [13] also answering Wang's question in the affirmative, proving that a properly edge-colored graph $G$ with at least $\left(\delta(G)^{2}+4 \delta(G)-4\right) / 4$ vertices has a rainbow matching of size $\delta(G)$.

The proof of Theorem 1 utilizes extremal techniques akin to those that appear in $[5,8,11]$ and [12]. We also implement a greedy algorithmic approach to demonstrate that it is possible to efficiently construct a rainbow matching of size $\delta$ in a properly edge-colored graph with minimum degree $\delta$ having order at least $6.5 \delta$. To our knowledge, an algorithmic approach of this type has not been previously employed in the study of rainbow matchings. 
Theorem 2. If $G$ is a properly edge-colored graph with minimum degree $\delta$ satisfying $|V(G)|>$ $\frac{13}{2} \delta-\frac{23}{2}+\frac{41}{8 \delta}$, then there is an $O\left(\delta(G)|V(G)|^{2}\right)$-time algorithm that produces a rainbow matching of size $\delta$ in $G$.

As a contrast, Itai, Rodeh, and Tanimota [4] proved that determining if an edge-colored graph $G$ has a rainbow matching of size $k$ is NP-complete, even if $G$ is bipartite. More recently, Le and Pfender [7] have shown that the problem of determining the maximum size of a rainbow matching in a properly edge-colored graph is NP-hard, even when restricted to properly edge-colored paths.

\section{Proof of Theorem 1}

Let $G$ be a properly edge-colored $n$-vertex graph with minimum degree $\delta$ and $n \geqslant 98 \delta / 23$. The theorem holds easily if $\delta \leqslant 2$, so we may assume that $\delta \geqslant 3$. By way of contradiction, let $G$ be a counterexample with $\delta$ minimized; thus $G$ does not contain a rainbow matching of size $\delta$. Further, we may assume that $|E(G)|$ is minimized, so in particular the vertices of degree greater than $\delta$ form an independent set, as otherwise we could delete an edge without lowering the minimum degree. We break the proof into a series of simple claims.

Let $\Delta(G)=d_{1} \geqslant d_{2} \geqslant \ldots \geqslant d_{n}=\delta$ with $d\left(v_{i}\right)=d_{i}$ be the degree sequence of $G$.

Lemma 3. For $1 \leqslant k \leqslant 2 \delta / 3$, there exists an $i \leqslant k$ such that $d_{i} \leqslant 3 \delta-k-2 i$.

Proof. Suppose that for some $k \leqslant 2 \delta / 3, d_{i} \geqslant 3 \delta+1-k-2 i$ for all $1 \leqslant i \leqslant k$. It follows that $d_{i}>\delta$ for $i \leqslant k$, and therefore $\left\{v_{1}, \ldots, v_{k}\right\}$ is an independent set. Delete the vertices $v_{1}, v_{2}, \ldots, v_{k}$ from $G$, and note that $\delta\left(G \backslash\left\{v_{1}, \ldots, v_{k}\right\}\right) \geqslant \delta-k$. By the minimality of $G$, there exists a rainbow matching $M_{k}$ of size $\delta-k$ in $G \backslash\left\{v_{1}, \ldots, v_{k}\right\}$.

The vertex $v_{k}$ has at most $2(\delta-k)$ neighbors in $M_{k}$, and is incident to at most $\delta-k$ edges colored with colors occurring in $M_{k}$. Thus, $v_{k}$ has a neighbor $w_{k} \notin V\left(M_{k}\right)$ such that the color of $v_{k} w_{k}$ does not occur in $M_{k}$, and we can extend $M_{k}$ by adding the edge $v_{k} w_{k}$; call the resulting rainbow matching $M_{k-1}$. Note that $w_{k} \neq v_{i}$ for $i \leqslant k$ as $\left\{v_{1}, \ldots v_{k}\right\}$ is an independent set.

The $j$ th iteration of this process produces a rainbow matching $M_{k-j}$ of size $\delta-k+j$ that contains $\left\{v_{k}, \ldots, v_{k-j+1}\right\}$. Hence $v_{k-j}$ has at most $2(\delta-k)+j$ neighbors in $M_{k-j}$ and is incident to at most $\delta-k+j$ edges colored with colors occurring in $M_{k-j}$. Thus there is a vertex $w_{k-j} \in N\left(v_{k-j}\right)$ such that the edge $v_{k-j} w_{k-j}$ extends $M_{k-j}$ to a $(\delta-k+j+1)$-edge rainbow matching $M_{k-(j+1)}$. Continuing in this fashion extends the matching $M_{k}$ by $k$ edges, yielding a rainbow matching of size $\delta$, a contradiction finishing the proof.

As a corollary of Lemma 3, we obtain the following.

Lemma 4. For $1 \leqslant k \leqslant 2 \delta / 3$, we have $\sum_{i=1}^{k} d_{i} \leqslant k(3 \delta-2-k)$, with equality only if $d_{1}=d_{k}=3 \delta-2-k$. 
Proof. We proceed by induction on $k$. For $k=1$, the statement follows from Lemma 3. Now let $k>1$ and let $i \leqslant k$ such that $d_{i} \leqslant 3 \delta-k-2 i$. By induction,

$$
\begin{gathered}
\sum_{j=1}^{i-1} d_{j} \leqslant(i-1)(3 \delta-1-i), \text { and } \\
\sum_{j=i}^{k} d_{j} \leqslant(k-i+1) d_{i} \leqslant(k-i+1)(3 \delta-k-2 i) .
\end{gathered}
$$

Thus,

$\sum_{j=1}^{k} d_{j} \leqslant(i-1)(3 \delta-1-i)+(k-i+1)(3 \delta-k-2 i)=3 k \delta-k^{2}-k+1-i(k+2-i) \leqslant k(3 \delta-2-k)$

and equality holds only if $i=1$ and $d_{1}=d_{k}=3 \delta-2-k$.

Let $C$ be a maximum color class in $G$ and let $|C|=a$. By the minimality of $G$, there exists a rainbow matching $M=\left\{x_{i} y_{i}: 1 \leqslant i \leqslant \delta-1\right\}$ of size $\delta-1$ in $G-C$. Without loss of generality, we may assume that $c\left(x_{i} y_{i}\right)=i$ for $1 \leqslant i \leqslant \delta-1$ and the edges in $C$ have color $\delta$. Let $W=V(G) \backslash V(M)$; observe that $|W|=n-2(\delta-1)$. If there is an edge $e$ in $G[W]$ with $c(e) \notin\{1, \ldots, \delta-1\}$ then we can add $e$ to $M$ to obtain a rainbow matching of size $\delta$. Thus we may assume that every edge whose color is not in $\{1, \ldots, \delta-1\}$ has an endpoint in $V(M)$. An edge $u v$ is good if its color is not in $\{1, \ldots, \delta-1\}$ and one of its endpoints is in $W$. A vertex $v \in V(M)$ is good if $v$ is incident with at least seven good edges.

Claim 5. For $i \in\{1, \ldots, \delta-1\}$, if $x_{i}$ is incident with at least three good edges, then no good edge is incident with $y_{i}$, and vice versa.

Proof. Suppose that $y_{i} u$ is a good edge. If $x_{i}$ is incident with at least three good edges, then $x$ has a neighbor $w$ such that $v w$ is a good edge, $w \neq u$, and $c\left(x_{i} w\right) \neq c\left(y_{i} u\right)$. Thus $\left(M \cup\left\{x_{i} w, y_{i} u\right\}\right) \backslash\left\{x_{i} y_{i}\right\}$ is a rainbow matching of size $\delta$, a contradiction.

By Claim 5, we may assume without loss of generality that $\left\{x_{1}, \ldots, x_{r}\right\}$ is the set of good vertices for some $r \geqslant 0$. Let $W^{\prime}=W \cup\left\{y_{1}, \ldots, y_{r}\right\}$.

Claim 6. No edge uv in $G\left[W^{\prime}\right]$ has color in $\{1, \ldots, r\}$.

Proof. By way of contradiction, assume that there is an edge $u v$ in $G\left[W^{\prime}\right]$ such that $c(u v) \in$ $\{1, \ldots, r\}$. Let $M^{\prime}$ be the subset of $M$ consisting of the edge with color $c(u v)$ and any edges with an endpoint in $\{u, v\}$. There are at most three such edges (the edge with color $c(u v)$ and possibly one for each endpoint); without loss of generality, let $M^{\prime}=\left\{x_{1} y_{1}, \ldots, x_{t} y_{t}\right\}$ (here $1 \leqslant t \leqslant 3$ ). Note that $x_{j}$ is a good vertex for $1 \leqslant j \leqslant t$. Thus there are distinct vertices $w_{1}, \ldots, w_{t}$ such that $x_{j} w_{j}$ is a good edge for $1 \leqslant j \leqslant t$ and the colors on the edges $u v, x_{1} w_{1}, \ldots, x_{t} w_{t}$ are distinct. Thus $\left(M \cup\left\{u v, x_{1} w_{1}, \ldots, x_{t} w_{t}\right\}\right) \backslash\left\{x_{1} y_{1}, \ldots, x_{t} y_{t}\right\}$ is a rainbow matching of size $\delta$, a contradiction. 
An edge $u v$ is nice if its color is not in $\{r+1, \ldots, \delta-1\}$ and one of its endpoints is in $W^{\prime}$. Note that every good edge is nice. Recall that every good edge has an endpoint in $V(M)$. By Claim 5 and Claim 6 , no nice edge lies in $G\left[W^{\prime}\right]$. Hence, every nice edge joins vertices in $W^{\prime}$ and $V(G) \backslash W^{\prime}$. A vertex $v \in V(M) \backslash\left\{x_{1}, \ldots, x_{r}, y_{1}, \ldots, y_{r}\right\}$ is nice if $v$ is incident with at least seven nice edges. Note that if there is no good vertex (i.e. $r=0$ ), then the definitions of good and nice vertices are the same and so there is also no nice vertex. Next, we prove analogues of Claim 5 and Claim 6 for nice vertices and edges.

Claim 7. For $i \in\{r+1, \ldots, \delta-1\}$, if $x_{i}$ is incident with at least three nice edges, then no nice edge is incident with $y_{i}$, and vice versa.

Proof. Suppose $y_{i} u$ is a nice edge for some $i \in\{r+1, \ldots, \delta-1\}$. If $x_{i}$ is incident to at least three nice edges, then $x_{i}$ has a neighbor $v$ such that $x_{i} v$ is a nice edge, $v \neq u$, and $c\left(x_{i} v\right) \neq$ $c\left(y_{i} u\right)$. Let $M^{\prime}$ be the subset of $M$ consisting of edges with an endpoint in $\{u, v\}$ or a color in $\left\{c\left(x_{i} v\right), c\left(y_{i} u\right)\right\}$. There are at most four such edges (possibly one with each endpoint and one with each color); without loss of generality, let $M^{\prime}=\left\{x_{1} y_{1}, \ldots, x_{t} y_{t}\right\}$ (here $0 \leqslant t \leqslant 4$ ). Note that $x_{j}$ is a good vertex for $1 \leqslant j \leqslant t$. Thus there are distinct vertices $w_{1}, \ldots, w_{t}$ such that $x_{j} w_{j}$ is a good edge for $1 \leqslant j \leqslant t$ and the colors on the edges $x_{i} v, y_{i} u, x_{1} w_{1}, \ldots, x_{t} w_{t}$ are distinct. Thus $\left(M \cup\left\{x_{i} v, y_{i} u, x_{1} w_{1}, \ldots, x_{t} w_{t}\right\}\right) \backslash\left\{x_{i} y_{i}, x_{1} y_{1}, \ldots, x_{t} y_{t}\right\}$ is a rainbow matching of size $\delta$, a contradiction.

By Claim 7, we may assume that $\left\{x_{r+1}, x_{r+2}, \ldots, x_{r+s}\right\}$ is the set of nice vertices for some $s \geqslant 0$.

Claim 8. No edge uv in $G\left[W^{\prime}\right]$ has color in $\{1, \ldots, r+s\}$.

Proof. By Claim 6, the claim holds if $s=0$. Assume that $s \geqslant 1$, and consequently $r \geqslant 1$. Without loss of generality, suppose that there is an edge $u v$ in $G\left[W^{\prime}\right]$ with $c(u w)=r+1$. Because $x_{r+1}$ is nice, it has a neighbor $v^{\prime}$ in $W^{\prime}$ such that $x_{r+1} v^{\prime}$ is a nice edge and $v^{\prime} \neq u, v$. Let $M^{\prime}$ be the subset of $M$ consisting of those edges an endpoint in $\left\{u, v, v^{\prime}\right\}$ or color $c\left(x_{r+1} v^{\prime}\right)$. Again there are at most four edges in $M^{\prime}$ and we let $M^{\prime}=\left\{x_{1} y_{1}, \ldots, x_{t} y_{t}\right\}$. Defining $w_{1}, \ldots, w_{t}$ as before, $\left(M \cup\left\{u v, x_{r+1} v^{\prime}, x_{1} w_{1}, \ldots, x_{t} w_{t}\right\}\right) \backslash\left\{x_{r+1} y_{r+1}, x_{1} y_{1}, \ldots, x_{t} y_{t}\right\}$ is a rainbow matching of size $\delta$, a contradiction.

Next, we count the number of nice edges in $G$.

Claim 9. There are at most $\max \{(3 \delta-8-r+s) r+6(\delta-1),(7 \delta / 3-7+s) r+6(\delta-1)\}$ nice edges in $G$.

Proof. Recall that $V(G) \backslash W^{\prime}=\left\{x_{1}, \ldots, x_{\delta-1}, y_{r+1}, \ldots, y_{\delta-1}\right\}$ and every nice edge joins vertices from $W^{\prime}$ and $V(G) \backslash W^{\prime}$. If $r \leqslant 2 \delta / 3$, then the set of good vertices is incident to at most $r(3 \delta-2-r)$ nice edges by Lemma 4 . Similarly, if $r \geqslant 2 \delta / 3$, then the set of good vertices is incident to at most $r(3 \delta-2-\lfloor 2 \delta / 3\rfloor) \leqslant r(7 \delta / 3-1)$ nice edges. For $i \in\{r+1, \ldots, r+s\}$, Claim 7 implies that $x_{i}$ is incident to at most $r+6$ nice edges and $y_{i}$ is incident to none. 
For $i \in\{r+s+1, \ldots, \delta-1\}$, by Claim 7 there are at most six nice edges with an endpoint in $\left\{x_{i}, y_{i}\right\}$. Therefore, the number of nice edges is at most

$$
(3 \delta-2-r) r+(r+6) s+6(\delta-1-r-s)=(3 \delta-8-r+s) r+6(\delta-1) \text { if } r \leqslant 2 \delta / 3,
$$

and

$$
(7 \delta / 3-1) r+(r+6) s+6(\delta-1-r-s)=(7 \delta / 3-7+s) r+6(\delta-1) \text { if } r \geqslant 2 \delta / 3 .
$$

Recall that $C$ is the color class with color $\delta,|C|=a$, and $C$ is a maximum size color class. Therefore there are at least $2(a-\delta+1)$ vertices in $W$ incident to an edge in $C$. Since every edge in $C$ has an endpoint in $V(M)$ it follows that there are at least $2(a-\delta+1)$ vertices in $V(M)$ joined to $W$ by edges in $C$. Without loss of generality, let $\{r+s+1, \ldots, r+s+t\}$ be the set of indices $i \in\{r+s+1, \ldots, \delta-1\}$ such that $x_{i}$ or $y_{i}$ is incident to an edge in $C$. By Claim 5 and Claim 7, we have

$$
t \geqslant a-\delta+1-\frac{r+s}{2} \text { and } r+s+t \leqslant \delta-1
$$

Claim 10. For $i \in\{r+s+1, \ldots, r+s+t\}$, there is at most one edge of color $i$ in $G[W]$.

Proof. Suppose $u v$ and $u^{\prime} v^{\prime}$ are edges of color $i$ in $G[W]$ for some $i \in\{r+s+1, \ldots, r+s+t\}$. Without loss of generality, we may assume that there exists $w \in W$ such that $c\left(x_{i} w\right)=\delta$ and $w \neq u, v$. Hence, $\left(M \cup\left\{u v, x_{i} w\right\}\right) \backslash\left\{x_{i} y_{i}\right\}$ is a rainbow matching of size $\delta$, a contradiction.

Now we count the number of nice edges from $W^{\prime}$ to $V(G) \backslash W^{\prime}$. Recall that each color class in $G$ contains at most $a$ edges. By Claim 8, there is no edge in $G\left[W^{\prime}\right]$ of color $i \in$ $\{r+1, \ldots, r+s\}$. Thus, for $i \in\{r+1, \ldots, r+s\}$ there are at most $a-1$ vertices in $W^{\prime}$ that are incident with an edge of color $i$. Since every color class has size at most $a$, for $i \in\{r+s+1, \ldots, \delta-1\}$ there are at most $2(a-1)$ vertices in $W^{\prime}$ that are incident with an edge of color $i$. Furthermore for $i \in\{r+s+1, \ldots, r+s+t\}$, Claim 10 implies that there are at most $a$ vertices in $W$ that are incident with an edge of color $i$. Since $W^{\prime} \backslash W=\left\{y_{1}, \ldots, y_{r}\right\}$, it follows that for $i \in\{r+s+1, \ldots, r+s+t\}$ there are at most $\min \{a+r, 2(a-1)\}$ vertices in $W^{\prime}$ that are incident with an edge of color $i$. It then follows, using the fact that $\left|W^{\prime}\right|=|W|+r=n-2(\delta-1)+r$ and (1), that the number of nice edges from $W^{\prime}$ to $V(G) \backslash W^{\prime}$ is at least

$$
\begin{aligned}
& \delta\left|W^{\prime}\right|-(a-1)(2 \delta-2-2 r-s-2 t)-\min \{a+r, 2(a-1)\} t \\
= & \delta n-2 \delta(\delta-1)+\delta r-(a-1)(2 \delta-2-2 r-s)+\max \{a-r-2,0\} t .
\end{aligned}
$$

We first consider the case when $r \leqslant 2 \delta / 3$. Applying the upper bound of $(3 \delta-8-r+$ $s) r+6(\delta-1)$ nice edges from Claim 9 , we obtain

$$
\delta n \leqslant(2 \delta-8-r+s) r+2(\delta+3)(\delta-1)+(a-1)(2 \delta-2-2 r-s)-\max \{a-r-2,0\} t .
$$


To finish the proof we bound the right hand side of (2) to obtain a contradiction. Note that the coefficient of $t$ is nonpositive. Thus, the right hand side of (2) is maximized when $t$ is minimized. By (1), $t \geqslant \max \{a-\delta+1-(r+s) / 2,0\}$.

If $a \leqslant \delta-1+(r+s) / 2$, then we let $t=0$. The coefficient of $a$ is nonnegative, and thus (2) is maximized when $a$ is maximized; hence we assume $a=\delta-1+(r+s) / 2$. Substituting for $a$ yields a negative quadratic in $s$ that is maximized when $s=1-r / 2$. Since $s$ is a nonnegative integer and $s=0$ if $r=0,(2)$ is maximized at $s=0$, which yields

$$
\delta n \leqslant 2(2 \delta+1)(\delta-1)+(\delta-5-2 r) r .
$$

This is maximized when $r=(\delta-5) / 4$, yielding $n \leqslant 33 \delta / 8-13 / 4+9 /(8 \delta)$, a contradiction.

If $a>\delta-1+r / 2$, we let $t=a-\delta+1-r / 2$. Since $t>0$, it follows that $r+s \leqslant \delta-2$. Thus $a-r-2>\delta-3-(r-s) / 2 \geqslant \delta-3-(\delta-2) / 2=\delta / 2-2$. As $\delta \geqslant 3$ and $a-r-2$ is an integer, $\max \{a-r-2,0\}=a-r-2$. Therefore the coefficient of $s$ in (2) is nonpositive and we may assume that $s=0$. Consequently, (2) becomes

$$
\delta n \leqslant(3 \delta-1-r / 2-a) a+(\delta-6-3 r / 2) r+2\left(\delta^{2}-1\right) .
$$

The right hand side is maximized when $a=3 \delta / 2-1 / 2-r / 4$, so

$$
\delta n \leqslant \frac{1}{16}\left(-28+68 \delta^{2}-24 \delta+4 \delta r-92 r-23 r^{2}\right),
$$

which is maximized when $r=2 \delta / 23-2$. This yields

$$
n \leqslant 98 \delta / 23-2+4 / \delta
$$

a contradiction.

To complete the proof of the theorem, we are left with the case $r \geqslant 2 \delta / 3$. Similarly to $(2)$, since we have at most $(7 \delta / 3-7+s) r+6(\delta-1)$ nice edges in $G$ by Claim 9 , we have

$$
\delta n \leqslant(4 \delta / 3-7+s) r+2(\delta+3)(\delta-1)+(a-1)(2 \delta-2-2 r-s)-\max \{a-r-2,0\} t .
$$

Again, the right hand side of (3) is maximized when $t$ is minimized.

If $a \leqslant \delta-1+(r+s) / 2$, then (3) is maximized when $t=0$ and $a=\delta-1+r / 2$. Again we may assume that $s=0$, yielding

$$
\delta n \leqslant-2+4 \delta^{2}-2 \delta+r(\delta / 3-4-r) .
$$

This is maximized when $r=\delta / 6-2$, yielding $n \leqslant 145 \delta / 36-8 / 3+6 / \delta$, a contradiction.

If $a>\delta-1+(r+s) / 2$, we let $t=a-\delta+1-(r+s) / 2$ and again we may assume that $s=0$. Then, (3) becomes

$$
\delta n \leqslant \frac{1}{6}\left(-6 a^{2}+3 a(6 \delta-2)+12 \delta^{2}-(3 a+30+3 r-2 \delta) r-12\right),
$$

which is maximized when $r$ is minimized. Since we have assumed that $r \geqslant 2 \delta / 3$, we have $r=2 \delta / 3$ and we are back in the case $r \leqslant 2 \delta / 3$, finishing the proof of the theorem. 


\section{Proof of Theorem 2}

We proceed by induction on $\delta(G)$. The result is trivial if $\delta(G)=1$. We assume that $G$ is a graph with minimum degree $\delta>1$ and order greater than $\frac{13}{2} \delta-\frac{23}{2}+\frac{41}{8 \delta}$.

Lemma 11. If $G$ has a color class containing at least $2 \delta-1$ edges, then $G$ has a rainbow matching of size $\delta$.

Proof. Let $C$ be a color class with at least $2 \delta-1$ edges. By induction, there is a rainbow matching $M$ of size $\delta-1$ in $G-C$. There are $2 \delta-2$ vertices covered by the edges in $M$, thus one of the edges in $C$ has no endpoint covered by $M$, and the matching can be extended.

It is also useful to note that we also have the following, which is identical to Lemma 3 when $k=1$.

Lemma 12. If $G$ satisfies $\Delta(G)>3 \delta-3$, then $G$ has a rainbow matching of size $\delta$.

We begin by preprocessing the graph so that each edge is incident to at least one vertex with degree $\delta$. To achieve this, arbitrarily order the edges in $G$ and process them in order. If both endpoints of an edge have degree greater than $\delta$ when it is processed, delete that edge. In the resulting graph, every edge is incident to a vertex with degree $\delta$. Furthermore, by Lemma 12 we may assume that $\Delta(G) \leqslant 3 \delta-3$; thus the degree sum of the endpoints of any edge is bounded above by $4 \delta-3$. After preprocessing, we begin the greedy algorithm.

In the $i$ th step of the algorithm, a smallest color class is chosen (without loss of generality, color $i$ ), and then an edge $e_{i}$ of color $i$ is chosen such that the degree sum of the endpoints is minimized. All the remaining edges of color $i$ and all edges incident with the endpoints of $e_{i}$ are deleted. The algorithm terminates when there are no edges in the graph.

We assume that the algorithm fails to produce a matching of size $\delta$ in $G$; suppose that the rainbow matching $M$ generated by the algorithm has size $k$. We let $R$ denote the set of vertices that are not covered by $M$.

Let $c_{i}$ denote the size of the smallest color class at step $i$. Since at most two edges of color $i+1$ are deleted in step $i$ (one at each endpoint of $e_{i}$ ), we observe that $c_{i+1}+2 \geqslant c_{i}$. Otherwise, at step $i$ color class $i+1$ has fewer edges. Let step $h$ be the last step in the algorithm in which a color class that does not appear in $M$ is completely removed from $G$. It then follows that $c_{h} \leqslant 2$, and in general $c_{i} \leqslant 2(h-i+1)$ for $i \in[h]$. Let $f_{i}$ denote the number of edges of color $i$ deleted in step $i$ with both endpoints in $R$. Since $f_{i}<c_{i}$, we have $f_{i} \leqslant 2(h-i)+1$ for $i \in[h]$. Note that after step $h$, there are exactly $k-h$ colors remaining in $G$. By Lemma 11, color classes contain at most $2 \delta-2$ edges, and therefore the last $k-h$ steps remove at most $(k-h)(2 \delta-2)$ edges. Furthermore, for $i>h$, the degree sum of the endpoints of $e_{i}$ is at most $2(\delta-1)$.

For $i \in[h]$, let $x_{i}$ and $y_{i}$ be the endpoints of $e_{i}$, and let $d_{i}(v)$ denote the degree of a vertex $v$ at the beginning of step $i$. Let $\mu_{i}=\max \left\{0, d_{i}\left(x_{i}\right)+d_{i}\left(y_{i}\right)-2 \delta\right\}$; note that $2 \delta \leqslant 2 \delta+\mu_{i} \leqslant 4 \delta-3$. Thus, at step $i$, at most $2 \delta+\mu_{i}+f_{i}-1$ edges are removed from the 
graph. Since the algorithm removes every edge from the graph, we conclude that

$$
|E(G)| \leqslant(k-h)(2 \delta-2)+\sum_{i=1}^{h}\left(2 \delta+\mu_{i}+f_{i}-1\right) .
$$

We now compute a lower bound for the number of edges in $G$. Since the degree sum of the endpoints of $e_{i}$ in $G$ is at least $2 \delta+\mu_{i}$, we immediately obtain the following inequality:

$$
\frac{n \delta+\sum_{i \in[h]} \mu_{i}}{2} \leqslant|E(G)| .
$$

If $f_{i}>0$ and $\mu_{i}>0$, then there is an edge with color $i$ having both endpoints in $R$. Since this edge was not chosen in step $i$ by the algorithm, the degree sum of its endpoints is at least $2 \delta+\mu_{i}$, and one of its endpoints has degree at least $\delta+\mu_{i}$. For each value of $i$ satisfying $f_{i}>0$, we wish to choose a representative vertex in $R$ with degree at least $\delta+\mu_{i}$. Since there are $f_{i}$ edges with color $i$ having both endpoints in $R$, there are $f_{i}$ possible representatives for color $i$. Since a vertex in $R$ with high degree may be the representative for multiple colors, we wish to select the largest system of distinct representatives.

Suppose that the largest system of distinct representatives has size $t$, and let $T$ be the set of indices of the colors that have representatives. For each color $i \in T$ there is a distinct vertex in $R$ with degree at least $\delta+\mu_{i}$. Thus we may increase the edge count of $G$ as follows:

$$
\frac{n \delta+\sum_{i \in[h]} \mu_{i}+\sum_{i \in T} \mu_{i}}{2} \leqslant|E(G)| .
$$

We let $\left\{f_{i}^{\downarrow}\right\}$ denote the sequence $\left\{f_{i}\right\}_{i \in[h]}$ sorted in nonincreasing order. Since $f_{i} \leqslant 2(h-$ $i)+1$, we conclude that $f_{i}^{\downarrow} \leqslant 2(h-i)+1$. Because there is no system of distinct representatives of size $t+1$, the sequence $\left\{f_{i}^{\downarrow}\right\}$ cannot majorize the sequence $\{t+1, t, t-1, \ldots, 1\}$. Hence there is a smallest value $p \in[t+1]$ such that $f_{p}^{\downarrow} \leqslant t+1-p$. Therefore, the maximum value of $\sum_{i=1}^{h} f_{i}^{\downarrow}$ is bounded by the sum of the sequence $\{2 h-1,2 h-3, \ldots, 2(h-p)+3, t+1-$ $p, \ldots, t+1-p\}$. Summing we attain

$$
\sum_{i \in[h]} f_{i} \leqslant(p-1)(2 h-p+1)+(h-p+1)(t+1-p) .
$$

Over $p$, this value is maximized when $p=t+1$, yielding $\sum_{i \in[h]} f_{i} \leqslant t(2 h-t)$. Since $h \leqslant \delta-1$, we then have $\sum_{i \in[h]} f_{i} \leqslant 2(\delta-1) t-t^{2}$.

We now combine bounds (4) and (5):

$$
\frac{n \delta+\sum_{i \in[h]} \mu_{i}+\sum_{i \in T} \mu_{i}}{2} \leqslant(k-h)(2 \delta-2)+\sum_{i=1}^{h}\left(2 \delta+\mu_{i}+f_{i}-1\right) .
$$


Hence, since $k \leqslant \delta-1$,

$$
\begin{aligned}
\frac{n \delta}{2} & \leqslant(2 \delta-1)(\delta-1)+\frac{1}{2} \sum_{[h] \backslash T} \mu_{i}+\sum_{i \in[h]} f_{i} \\
& \leqslant(2 \delta-1)(\delta-1)+(\delta-1-t)(\delta-3 / 2)+2(\delta-1) t-t^{2} \\
& \leqslant 3 \delta^{2}-\frac{11}{2} \delta+\frac{5}{2}+\left(\delta-\frac{1}{2}\right) t-t^{2} .
\end{aligned}
$$

This bound is maximized when $t=\left(\delta-\frac{1}{2}\right) / 2$. Thus

$$
n \leqslant \frac{13}{2} \delta-\frac{23}{2}+\frac{41}{8 \delta}
$$

contradicting our choice for the order of $G$.

It remains to show that this proof provides the framework of a $O\left(\delta(G)|V(G)|^{2}\right)$-time algorithm that generates a rainbow matching of size $\delta(G)$ in a properly edge-colored graph $G$ of order at least $\frac{13}{2} \delta-\frac{23}{2}+\frac{41}{8 \delta}$. Given such a $G$, we create a sequence of graphs $\left\{G_{i}\right\}$ as follows, letting $G=G_{0}, \delta=\delta(G)$, and $n=|V(G)|$. First, determine $\delta\left(G_{i}\right), \Delta\left(G_{i}\right)$, and the maximum size of a color class in $G_{i}$; this process takes $O\left(n^{2}\right)$-time. If $\Delta\left(G_{i}\right) \leqslant 3 \delta\left(G_{i}\right)-3$ and the maximum color class has at most $2 \delta\left(G_{i}\right)-2$ edges, then terminate the sequence and set $G_{i}=G^{\prime}$. If $\Delta\left(G_{i}\right)>3 \delta\left(G_{i}\right)-3$, then delete a vertex $v$ of maximum degree and then process the edges of $G_{i}-v$, iteratively deleting those with two endpoints of degree at least $\delta\left(G_{i}\right)$; the resulting graph is $G_{i+1}$. If $\Delta\left(G_{i}\right) \leqslant 3 \delta\left(G_{i}\right)-3$ but a maximum color class $C$ has at least $2 \delta\left(G_{i}\right)-1$ edges, then delete $C$ and then process the edges of $G_{i}-C$, iteratively deleting those with two endpoints of degree at least $\delta\left(G_{i}\right)$; the resulting graph is $G_{i+1}$. Note that $\delta\left(G_{i+1}\right)=\delta\left(G_{i}\right)-1$. If this process generates $G_{\delta}$, we set $G^{\prime}=G_{\delta}$ and terminate. Generating the sequence $\left\{G_{i}\right\}$ consists of at most $\delta$ steps, each taking $O\left(n^{2}\right)$-time.

Given that $G^{\prime}=G_{i}$, the algorithm from the proof of Theorem 2 takes $O\left(\delta n^{2}\right)$-time to generate a matching of size $\delta-i$ in $G^{\prime}$. The preprocessing step and the process of determining a smallest color class and choosing an edge in that class whose endpoints have minimum degree sum both take $O\left(n^{2}\right)$-time. This process is repeated at most $\delta$ times.

A matching of size $\delta-(i+1)$ in $G_{i+1}$ is easily extended in $G_{i}$ to a matching of size $\delta-i$ using the vertex of maximum degree or maximum color class. The process of extending the matching takes $O(\delta)$-time. Thus the total run-time of the algorithm generating the rainbow matching of size $\delta$ in $G$ is $O\left(\delta n^{2}\right)$.

It is worth noting that the analysis of the greedy algorithm used in the proof of Theorem 2 could be improved. In particular, the bound $c_{i+1} \geqslant c_{i}-2$ is sharp only if at step $i$ there are an equal number of edges of color $i$ and $i+1$ and both endpoints of $e_{i}$ are incident to edges with color $i+1$. However, since one of the endpoints of $e_{i}$ has degree at most $\delta$, at most $\delta-1$ color classes can lose two edges in step $i$. Since the maximum size of a color class in $G$ is at most $2 \delta-2$, if $G$ has order at least $6 \delta$, then there are at least $3 \delta / 2$ color classes. Thus, for small values of $i$, the bound $c_{i} \leqslant 2(k-i+1)$ can likely be improved. However, we doubt that such analysis of this algorithm can be improved to yield a bound on $|V(G)|$ better than $6 \delta$. 


\section{References}

[1] R. A. Brualdi and H. J. Ryser, Combinatorial Matrix Theory, Cambridge University Press, Cambridge, UK, 1991.

[2] R. Diestel, Graph Theory, Springer-Verlag, New York, 1997.

[3] G. A. Dirac, Some theorems on abstract graphs, Proc. London Math. Soc. (3) 2 (1952), 69-81.

[4] A. Itai, M. Rodeh, and S. Tanimoto, Some matching problems for bipartite graphs, J. Assoc. Comput. Mach. 25 (1978), no. 4, 517-525.

[5] A. Kostochka and M. Yancey, Large rainbow matchings in edge-colored graphs, In Preparation.

[6] M. Kano and X. Li, Monochromatic and heterochromatic subgraphs in edge-colored graphs - a survey, Graphs Comb. 24 (2008), 237-263.

[7] V. B. Le and F. Pfender, Complexity results for rainbow matchings, In Preparation.

[8] T. D. LeSaulnier, C. Stocker, P. S. Wenger, and D. B. West, Rainbow matching in edge-colored graphs, Electron. J. Combin. 17 (2010), \#N26.

[9] H. J. Ryser, Neuere Probleme der Kombinatorik, in "Vorträge über Kombinatorik Oberwolfach", Mathematisches Forschungsinstitut Oberwolfach, July 1967, 24-29.

[10] S. K. Stein, Transversals of Latin squares and their generalizations, Pacific J. Math. 59 (1975), no. 2, 567-575.

[11] G. Wang, Rainbow matchings in properly edge colored graphs, Electron. J. Combin. 18 (2011), \#P162.

[12] G. Wang and H. Li, Heterochromatic matchings in edge-colored graphs, Electron. J. Combin. 15 (2008), \#R138.

[13] G. Wang, J. Zhang, and G. Liu, Existence of rainbow matchings in properly edge-colored graphs, Front. Math. China 7 (2012), no. 3, 543-550.

[14] I. M. Wanless, Transversals in Latin squares: a survey, Surveys in Combinatorics 2011, London Math. Soc. Lecture Note Series 392 (2011), 403-437. 